\title{
Tilted Bianchi Type IX Dust Fluid Cosmological Model in General Relativity
}

\author{
Anita Bagora (Menaria) \\ Department of Mathematics, Jaipur National University, Rajasthon, Jaipur 302025, India \\ Correspondence should be addressed to Anita Bagora (Menaria), anita_bagora@yahoo.com
}

Received 21 October 2012; Accepted 8 November 2012

Academic Editors: R. N. Henriksen and P. A. Hughes

Copyright ( $) 2012$ Anita Bagora (Menaria). This is an open access article distributed under the Creative Commons Attribution License, which permits unrestricted use, distribution, and reproduction in any medium, provided the original work is properly cited.

Some tilted Bianchi type IX dust fluid cosmological model is investigated. To get a deterministic model we assume that $a=b^{1 / 2}$, where $a$ and $b$ are metric potentials and the fluid is pressureless, that is, $p=0$. Some physical and geometric properties of the model are discussed. Also, we have discussed a special model in terms of $t$ only.

\section{Introduction}

Bianchi type IX cosmological models are interesting because these models allow not only expansion but also rotation and shear, and in general these are anisotropic. Many relativists have taken keen interest in studying Bianchi type IX universe because familiar solutions like Robertson-Walker universe, the de Sitter universe, the Taub-Nut solutions, and so forth are of Bianchi type IX space-times. Bianchi type IX universe includes closed FRW models. The homogeneous and isotropic FRW cosmological models, which are used to describe standard cosmological models, are particular cases of Bianchi type I, V and IX space-times according to the constant curvature of the physical three-space, $t=$ constant, that is zero, negative or positive. In these models, neutrino viscosity explains the large radiation entropy in the universe and the degree of isotropy of the cosmic background radiation. The standard cosmological models are too restrictive because of the insistence on the isotropy of the physical three spaces; several attempts have been made to study nonstandard cosmological models [1-3]. It is therefore interesting to carry out the detailed studies of gravitational fields, which are described by space-time of various Bianchi types. Vaidya and Patel [4] have studied spatially homogeneous space time of Bianchi type IX, and they have outlined a general scheme for the derivation of exact solutions of Einstein's field equations in the presence of perfect fluid and pure radiation fields. Krori et al. [5] and Chakraborty and Nandy [6] have derived cosmological models of Bianchi type II, VIII, and IX. These and many other researchers namely Uggla and Zur-Muhlen [7], Burd et al. [8], King [9], and Paternoga and Graham et al. [10] have studied Bianchi type IX space-time in different context. Bali and Yadav [11] have investigated Bianchi type IX viscous fluid cosmological models. Pradhan et al. [12] derived Bianchi type IX viscous fluid cosmological models with a varying cosmological constant.

There has been a considerable interest in spatially homogeneous and anisotropic cosmological models in which the fluid flow is not normal to the hypersurface of homogeneity. These are called tilted universes. The general dynamics of tilted cosmological models have been studied by King and Ellis [13], Ellis and King [14]. Bradley and Sviestins [15] have shown that heat flow is expected for tilted universes. Mukherjee [16] has investigated Bianchi type I cosmological model with heat flux in general relativity. The tilted cosmological models with heat flux have been investigated by a number of authors namely Banerjee and Sanyal [17], Coley [18], Roy and Prasad [19], Roy and Banerjee [20]. Bagora [21, 22] have also investigated Bianchi type I and III tilted cosmological model in a different context.

In general relativity, a dust solution is an exact solution of the Einstein field equation in which the gravitational field is produced entirely by the mass, momentum, and stress density of a perfect fluid which has positive mass energy density but vanishing pressure. Concerning the tilted perfect fluid models, Bradley [23] obtained all tilted and expanding dust 
self-similar cosmologies that are hypersurface homogeneous. Carr [24] classified spherically symmetric self similar dust models. Bagora et al. [25, 26] have investigated tilted dust magnetic cosmological models.

Motivated by the situations discussed above, in this paper we shall focus on the problem tilted Bianchi type IX cosmological models with dust fluid, and we investigated dust cosmological model. To get the deterministic model, we have assumed supplementary condition $a=b^{1 / 2}$, where $a$ and $b$ are metric potentials. The all physical aspects of the model are discussed. Also, we discussed special model in terms of " $t$ ".

\section{Field Equations and Solutions}

We consider the homogeneous anisotropic Bianchi type IX metric in the following form:

$$
\begin{aligned}
d s^{2}= & -d t^{2}+a^{2} d x^{2}+b^{2} d y^{2} \\
& +\left(b^{2} \sin ^{2} y+a^{2} \cos ^{2} y\right) d z^{2}-2 a^{2} \cos y d x d z,
\end{aligned}
$$

where $a$ and $b$ are functions of cosmic time " $t$ " alone.

The energy-momentum tensor for perfect fluid distribution with heat conduction is given by Ellis [27] as

$$
T_{i j}=(\in+p) v_{i} \nu^{j}+p g_{i}^{j}+q_{i} \nu^{j}+v_{i} q^{j},
$$

together with

$$
\begin{gathered}
g_{i j} \nu^{i} \nu^{j}=-1, \\
q_{i} q^{j}>0, \\
q_{i} \nu^{i}=0 .
\end{gathered}
$$

Here $p$ is the pressure and $\in$ the density and $q_{i}$ the heat conduction vector orthogonal to $v^{i}$.

The fluid flow vector $v^{i}$ has the components $(\sinh \lambda / a$, $0,0, \cosh \lambda)$ satisfying the condition (3) and $\lambda$ is the tilt angle.

The Einstein's field equation

$$
R_{i}^{j}-\frac{1}{2} R g_{i}^{j}=-8 \pi T_{i}^{j}, \quad(\Lambda=0, c=G=1) .
$$

The field equation for the line element (1) leads to

$$
\begin{aligned}
& \frac{2 \ddot{b}}{b}+\frac{\dot{b}^{2}}{b^{2}}-\frac{3}{4} \frac{a^{2}}{b^{4}}+\frac{1}{b^{2}}-8 \pi\left[(\in+p) \sinh ^{2} \lambda+p+2 q_{1} \frac{\sinh \lambda}{a}\right], \\
&= \frac{\ddot{a}}{a}+\frac{\ddot{b}}{b}+\frac{\dot{a} \dot{b}}{a b}+\frac{a^{2}}{4 b^{4}}=-8 \pi p, \\
& \frac{2 \dot{a} \dot{b}}{a b}+ \frac{\dot{b}^{2}}{b^{2}}+\frac{1}{b^{2}}-\frac{a^{2}}{4 b^{4}} \\
&= 8 \pi\left[(\in+p) \cosh { }^{2} \lambda-p+2 q_{1} \frac{\sinh \lambda}{a}\right], \\
&(\in+p) a \sinh \lambda \cosh \lambda+q_{1} \cosh \lambda+q_{1} \frac{\sinh \cosh ^{2} \lambda}{\cosh \lambda}=0,
\end{aligned}
$$

where $(\cdot)$ denotes the ordinary differentiation with respect to cosmic time " $t$ ".

\section{Solutions of Field Equations}

Equations from (7) to (10) are four equations in six unknown $a, b, \in, p, \lambda$, and $q_{1}$. Thus we require two more conditions to determine complete solution.

Firstly we assume that the model is filled with dust of perfect fluid which leads to

$$
P=0
$$

Secondly, we assumed relation between metric potentials as

$$
a=b^{1 / 2} .
$$

The motive behind assuming the conditions $a=b^{1 / 2}$ is explained as follows. Referring to Throne [28], the observations of velocity-redshift relation for extra-galactic sources suggest that the Hubble expansion of the universe is isotropic to within $30 \%[29,30]$. More precisely, the redshift studies place the limit $\sigma / H \leq 0.30$, where $\sigma$ is the shear and $H$ the Hubble constant. Collins et al. [31] have pointed out that for spatially homogeneous metric, the normal congruence to the homogeneous hypersurface satisfies the condition $\sigma / \theta=$ constant. The condition $\sigma_{1}^{1} / \theta=$ constant leads to $a=b^{1 / 2}$ when the tilt angle $\lambda=0$. Here $\sigma_{1}^{1}$ is the eigenvalue of shear tensor $\sigma_{i}^{j}$.

Equations (7) and (9) lead to

$$
\frac{2 \ddot{b}}{b}+\frac{2 \dot{b}^{2}}{b^{2}}+\frac{2 \dot{a} \dot{b}}{a b}+\frac{2}{b^{2}}-\frac{a^{2}}{b^{4}}=8 \pi(\in-p) .
$$

Using (11) in (13), we have

$$
\frac{2 \ddot{b}}{b}+\frac{2 \dot{b}^{2}}{b^{2}}+\frac{2 \dot{a} \dot{b}}{a b}+\frac{2}{b^{2}}-\frac{a^{2}}{b^{4}}=8 \pi \in .
$$

Again from equations (7) and (9), we have

$$
\frac{\ddot{a}}{a}+\frac{\ddot{b}}{b}+\frac{\dot{a} \dot{b}}{a b}+\frac{a^{2}}{2 b^{4}}=0 .
$$

Using condition (12) in (15), we have

$$
\frac{3 \ddot{b}}{2 b}+\frac{1}{4}\left(\frac{\dot{b}}{b}\right)^{2}=\frac{-1}{4 b^{3}} .
$$

This leads to

$$
2 f f^{\prime}+\frac{f^{2}}{3 b}=-\frac{1}{3 b^{2}},
$$

where $\dot{b}=f(b)$ and $\ddot{b}=f f^{\prime}$.

Equation (17) leads to

$$
\begin{gathered}
\dot{b}=\sqrt{\frac{2 K b^{2 / 3}+1}{2 b},} \\
\ddot{b}=\frac{-\left(2 K b^{2 / 3}+1\right)}{12 b^{2}},
\end{gathered}
$$

where " $K$ " is constant of integration. 
The metric (1) reduces to the following form:

$$
\begin{aligned}
d S^{2}= & \frac{-2 T d T^{2}}{\left(2 T^{2 / 3} K+1\right)}+T d X^{2}+T^{2} d Y^{2} \\
& +\left(T^{2} \sin ^{2} Y+T \cos ^{2} Y\right) d Z^{2}-2 T \cos Y d Z d X
\end{aligned}
$$

where $b=T, x=X, y=Y, z=Z$.

\section{Some Physical and Geometrical Properties}

The density for the model (19) is given by

$$
8 \pi \in=\frac{8 K T^{2 / 3}+6 T}{3 T^{3}} .
$$

The tilt angle $\lambda$ is given by

$$
\begin{aligned}
& \cosh \lambda=\sqrt{\frac{3\left(8 K T^{2 / 3}+4 T+3\right)}{2\left(8 K T^{2 / 3}+9\right)}}, \\
& \sinh \lambda=\sqrt{\frac{\left(8 K T^{2 / 3}+12 T-9\right)}{2\left(8 K T^{2 / 3}+9\right)}} .
\end{aligned}
$$

The scalar expansion $\theta$ calculated for the flow vector $v^{i}$ is given by

$$
\theta=\frac{\psi_{1}}{2 T} \sqrt{\frac{\left(2 K T^{2 / 3}+1\right)}{3\left(8 K T^{2 / 3}+4 T+3\right)\left(8 K T^{2 / 3}+9\right)^{3}}} .
$$

The nonvanishing components of shear tensor $\left(\sigma_{i j}\right)$ and rotation tensor $\left(\omega_{i j}\right)$ are given by

$$
\begin{aligned}
& \sigma_{11}= \frac{\psi_{2}}{4} \sqrt{\frac{\left(2 K T^{2 / 3}+1\right)\left(8 K T^{2 / 3}+4 T+3\right)}{3 T\left(8 K T^{2 / 3}+9\right)^{5}}}, \\
& \sigma_{22}= \frac{\psi_{3}}{12} \sqrt{\frac{T\left(2 K T^{2 / 3}+1\right)}{3\left(8 K T^{2 / 3}+4 T+3\right)\left(8 K T^{2 / 3}+9\right)^{3}}}, \\
& \sigma_{33}= \frac{\left[\psi_{4} \cos ^{2} y+T \psi_{5} \sin ^{2} y\right]}{12} \\
& \times \sqrt{\frac{\left(2 K T^{2 / 3}+1\right)}{3 T\left(8 K T^{2 / 3}+4 T+3\right)\left(8 K T^{2 / 3}+9\right)^{3}}}, \\
& \sigma_{44}= \frac{\left(8 K T^{2 / 3}+12 T-9\right) \psi_{6}}{12 T} \\
& \times \sqrt{\frac{2 K T^{2 / 3}+1}{3 T\left(8 K T^{2 / 3}+4 T+3\right)\left(8 K T^{2 / 3}+9\right)^{5}}}, \\
& \sigma_{14}= \frac{-\psi_{2}}{12 T} \sqrt{\frac{\left(2 K T^{2 / 3}+1\right)\left(8 K T^{2 / 3}+12 T-9\right)}{\left(8 K T^{2 / 3}+9\right)^{5}}}, \\
& \frac{\left(2 K T^{2 / 3}+1\right)\left(8 K T^{2 / 3}+12 T-9\right)}{2\left(8 K T^{2 / 3}+9\right)}
\end{aligned}
$$

Here

$$
\begin{gathered}
\psi_{1}=384 K^{2} T^{4 / 3}+200 K T^{5 / 3}+243 T+600 K T^{2 / 3}+162, \\
\psi_{2}=192 K^{2} T^{4 / 3}+64 K T^{5 / 3}+192 K T^{2 / 3}+81 \\
\psi_{3}=192 K^{2} T^{4 / 3}+64 K T^{5 / 3}+108 T+192 K T^{2 / 3}-27, \\
\psi_{4}=384 K^{2} T^{4 / 3}+160 K T^{5 / 3}+108 T+480 K T^{2 / 3}, \\
\psi_{5}=960 K^{2} T^{4 / 3}+448 K T^{5 / 3}+432 T+1344 K T^{2 / 3}+405, \\
\psi_{6}=192 K^{2} T^{4 / 3}-64 K T^{5 / 3}+192 K T^{2 / 3}+81 .
\end{gathered}
$$

The flow vectors $v^{i}$ and heat conduction vectors $q_{i}$ for the model (19) are given by

$$
\begin{gathered}
v^{1}=\sqrt{\frac{\left(8 K T^{2 / 3}+12 T-9\right)}{2 T\left(8 K T^{2 / 3}+9\right)}}, \\
v^{4}=\sqrt{\frac{3\left(8 K T^{2 / 3}+4 T+3\right)}{2\left(8 K T^{2 / 3}+9\right)}}, \\
q_{1}=\frac{-\left(8 K T^{2 / 3}+4 T+3\right)}{2 \pi T^{3}} \sqrt{\frac{8 K T^{2 / 3}+12 T+9}{2\left(8 K T^{2 / 3}+9\right)}} \\
q_{4}=\frac{\left(8 K T^{2 / 3}+12 T-9\right)}{6 \pi T^{7 / 2}} \sqrt{\frac{3\left(8 K T^{2 / 3}+4 T+3\right)}{2\left(8 K T^{2 / 3}+9\right)}} .
\end{gathered}
$$

The rates of expansion $H_{i}$ in the direction of $x, y$, and $z$ axes are given by

$$
\begin{aligned}
& H_{1}=\frac{1}{4 T^{3 / 2}} \sqrt{4 K T^{2 / 3}+2} \\
& H_{2}=\frac{1}{2 T^{3 / 2}} \sqrt{4 K T^{2 / 3}+2} \\
& H_{3}=\frac{1}{2 T^{3 / 2}} \sqrt{4 K T^{2 / 3}+2}
\end{aligned}
$$

4.1. Special Model I. To find model in terms of $t$, we assume the constant $K=0$. Then, we have

$$
b=\frac{3\left(t+k_{1}\right)^{2 / 3}}{2}
$$

where " $k_{1}$ " is constant of integration.

Then metric (1) reduces in the following form:

$$
\begin{aligned}
d s^{2}= & -d \tau^{2}+\frac{3}{4} \tau^{2 / 3}\left\{d x^{2}+\frac{3}{4} \tau^{2 / 3} d y^{2}-2 \cos y d x d z\right\} \\
& +\frac{3}{4} \tau^{2 / 3}\left\{\frac{3}{4} \tau^{2 / 3} \sin ^{2} y+\cos y\right\} d z^{2} .
\end{aligned}
$$

The density for the model (28) is given by

$$
8 \pi \in=\frac{2}{9 \tau^{4 / 3}} .
$$


The tilt angle $\lambda$ is given by

$$
\begin{aligned}
& \cosh \lambda=\sqrt{\frac{\tau^{2 / 3}+1}{2},} \\
& \sinh \lambda=\sqrt{\frac{\tau^{2 / 3}-1}{2} .}
\end{aligned}
$$

The scalar expansion $\theta$ calculated for the flow vector $v^{i}$ is given by

$$
\theta=\frac{2\left(162 \tau^{2 / 3}+135\right)}{81 \tau \sqrt{3\left(\tau^{2 / 3}+1\right)}}
$$

The flow vectors $v^{i}$ and heat conduction vectors $q_{i}$ for the model (28) are given by

$$
\begin{gathered}
v^{1}=\frac{\sqrt{\tau^{2 / 3}-1}}{\tau^{1 / 3}}, \\
v^{4}=\sqrt{\frac{\tau^{2 / 3}+1}{2}}, \\
q_{1}=\frac{-\left(\tau^{2 / 3}+1\right) \sqrt{\tau^{2 / 3}-1}}{144 \pi T^{5 / 3}}, \\
q_{4}=\frac{\left(\tau^{2 / 3}-1\right) \sqrt{2\left(\tau^{2 / 3}+1\right)}}{144 \pi \tau^{2}} .
\end{gathered}
$$

The nonvanishing components of shear tensor $\left(\sigma_{i j}\right)$ and rotation tensor $\left(\omega_{i j}\right)$ are given by

$$
\begin{aligned}
& \sigma_{11}=\frac{\left(3 \tau^{2 / 3}+1\right)}{4 \tau^{1 / 3}} \sqrt{\frac{\tau^{2 / 3}+1}{3},} \\
& \sigma_{22}=\frac{\left(3 \tau^{2 / 3}-1\right)}{24 \tau^{1 / 3}} \sqrt{\frac{1}{3\left(\tau^{2 / 3}+1\right)}}, \\
& \sigma_{44}=\frac{\left(\tau^{2 / 3}-1\right)}{9 \tau} \sqrt{\frac{1}{3\left(\tau^{2 / 3}+1\right)}}, \\
& \sigma_{14}=\frac{2\left(\tau^{2 / 3}+3\right)}{9 \tau^{2 / 3}} \sqrt{\frac{3\left(\tau^{2 / 3}+1\right)}{3}}, \\
& \omega_{14}=\frac{1}{6 \tau^{1 / 3}} \sqrt{\frac{3\left(\tau^{2 / 3}-1\right)}{2}} .
\end{aligned}
$$

The rates of expansion $H_{i}$ in the direction of $x, y$, and $z$ axes are given by

$$
\begin{aligned}
& H_{1}=\frac{1}{3 \tau}, \\
& H_{2}=\frac{2}{3 \tau}, \\
& H_{3}=\frac{2}{3 \tau} .
\end{aligned}
$$

\section{Conclusions}

The model (19) has point-type singularity at $T=0$. The model starts to expand with the big bang at $T=0$ and stops at $T=\infty$. The model is expanding, shearing, rotating and anisotropic in general. Since ${ }_{T \rightarrow \infty}^{L t}(\sigma / \theta) \neq 0$ then the model does not approach isotropy for large values of $T$. The heat conduction vectors $q^{1} \rightarrow \infty$, and $q^{4} \rightarrow \infty$ at initial stage whereas as $T=\infty, q^{1} \rightarrow 0$, and $q^{4} \rightarrow 0$. The $x, y$ and $z$ components of Hubble parameters $H_{i} \rightarrow \infty, T \rightarrow 0$ and $H_{i} \rightarrow$ 0 as $T \rightarrow \infty$. Therefore, these are decreasing function of time.

In the model (28), initially the rate of expansion $\theta$ is infinite but for the large time as $\tau \rightarrow \infty$ the expansion will be completely vanished. The components of Hubble parameters are well behaved at $\tau>1$ and will become zero for large time.

For particularly at $\tau=\tau_{0}, H_{1}, H_{2}, H_{3}, \theta, \sigma^{2}$ and $\in$ are all infinite and the volume is zero. This shows that model has finite singularity at $\tau=\tau_{0}$. Therefore, this model starts expanding with power law expansion, and it has finite physical singularity.

\section{References}

[1] M. A. H. MacCallum, "Anisotropic and inhomogeneous relativistic cosmologies," in General Relativity: An Einstein Centenary Survey, S. W. Hawking and W. Israel, Eds., pp. 533-580, Cambridge University Press, 1979.

[2] J. V. Narlikar, Introduction to Cosmology, Jones and Bertlett Publications, 1983.

[3] J. V. Narlikar and A. K. Kembhavi, Fundamental of Cosmic Physics, vol. 6, Jones and Bartlett Publications, 1980.

[4] P. C. Vaidya and L. K. Patel, "Gravitational fields with spacetimes of Bianchi type IX," Pramana, vol. 27, no. 1-2, pp. 63-72, 1986.

[5] K. D. Krori, T. Chaudhury, C. R. Mahanta, and A. Mazumdar, "Some exact solutions in string cosmology," General Relativity and Gravitation, vol. 22, no. 2, pp. 123-130, 1990.

[6] S. Chakraborty and G. C. Nandy, "Cosmological studies in Bianchi II, VIII space-time," Astrophysics and Space Science, vol. 198, no. 2, pp. 299-308, 1992.

[7] C. Uggla and H. Zur-Muhlen, "Compactified and reduced dynamics for locally rotationally symmetric Bianchi type IX perfect fluid models," Classical and Quantum Gravity, vol. 7, no. 8, pp. 1365-1385, 1990.

[8] A. B. Burd, N. Buric, and G. F. R. Ellis, "A numerical analysis of chaotic behaviour in Bianchi IX models," General Relativity and Gravitation, vol. 22, no. 3, pp. 349-363, 1990.

[9] D. H. King, "Gravity-wave insights to bianchi type-IX universes," Physical Review D, vol. 44, no. 8, pp. 2356-2368, 1991.

[10] R. Paternoga and R. Graham, "Exact quantum states for the diagonal Bianchi type IX model with negative cosmological constant," Physical Review D, vol. 54, no. 8, pp. 4805-4812, 1996.

[11] R. Bali and M. K. Yadav, "Bianchi Type-IX viscous fluid cosmological model in general relativity," Pramana, vol. 64, no. 2, pp. 187-196, 2005.

[12] A. Pradhan, S. K. Srivastav, and M. K. Yadav, "Some homogeneous Bianchi type IX viscous fluid cosmological models with a varying $\Lambda$," Astrophysics and Space Science, vol. 298, pp. 419432, 2005.

[13] A. R. King and G. F. R. Ellis, "Tilted homogeneous cosmological models," Communications in Mathematical Physics, vol. 31, no. 3, pp. 209-242, 1973. 
[14] G. F. R. Ellis and A. R. King, "Was the big bang a whimper?" Communications in Mathematical Physics, vol. 38, no. 2, pp. 119-156, 1974.

[15] J. M. Bradley and E. Sviestins, "Some rotating, time-dependent Bianchi type VIII cosmologies with heat flow," General Relativity and Gravitation, vol. 16, no. 12, pp. 1119-1133, 1984.

[16] G. Mukherjee, “A Bianchi type I tilted universe," Journal of Astrophysics and Astronomy, vol. 4, no. 4, pp. 295-300, 1983.

[17] A. Banerjee and A. K. Sanyal, "Irrotational Bianchi V viscous fluid cosmology with heat flux," General Relativity and Gravitation, vol. 20, no. 2, pp. 103-113, 1988.

[18] A. A. Coley, "Bianchi V imperfect fluid cosmology," General Relativity and Gravitation, vol. 22, no. 1, pp. 3-18, 1990.

[19] S. R. Roy and A. Prasad, "Some L.R.S. Bianchi type V cosmological models of local embedding class one," General Relativity and Gravitation, vol. 26, no. 10, pp. 939-950, 1994.

[20] S. R. Roy and S. K. Banerjee, "Bianchi $\mathrm{VI}_{0}$ electric type cosmological models in general relativity with stiff fluid and heat conduction," General Relativity and Gravitation, vol. 28, no. 1, pp. 27-33, 1996.

[21] A. Bagora, "A tilted homogeneous cosmological model with disordered radiations and heat conduction in presence of magnetic field," Electronic Journal of Theoretical Physics, vol. 7, no. 24, pp. 373-382, 2010.

[22] A. Bagora, "Bianchi Type-III stiff fluid cosmological model in general relativity," Astrophysics and Space Science, vol. 319, no. 2-4, pp. 155-159, 2009.

[23] M. Bradley, "Dust EPL cosmologies," Classical and Quantum Gravity, vol. 5, p. L15, 1988.

[24] B. J. Carr, "Classification of spherically symmetric self-similar dust models," Physical Review D, vol. 4, Article ID 044022, 2000.

[25] A. Bagora, G. S. Rathore, and P. Bagora, "Tilted Bianchi Type I dust fluid magnetized cosmological model in general relativity," Turkish Journal of Physics, vol. 33, no. 3, pp. 167-177, 2009.

[26] G. S. Rathore and A. Bagora, "Magnetized dust fluid tilted universe for perfect fluid distribution in general relativity," Advanced Studies in Theoretical Physics, vol. 2, no. 17, pp. 817824, 2008.

[27] G. F. R. Ellis, General Relativity and Cosmology, Academic Press, New York, NY, USA, 2008.

[28] K. S. Thorne, "Primordial element formation, primordial magnetic fields, and the isotropy of the universe," Astrophysical Journal, vol. 148, pp. 51-68, 1967.

[29] R. Kantowski and R. K. Sachs, "Some spatially homogeneous anisotropic relativistic cosmological models," Journal of Mathematical Physics, vol. 7, no. 3, pp. 443-446, 1966.

[30] J. Kristian and R. K. Sachs, "Observations in Cosmology," Astrophysical Journal, vol. 143, pp. 379-399, 1966.

[31] C. B. Collins, E. N. Glass, and D. A. Wilkinson, "Exact spatially homogeneous cosmologies," General Relativity and Gravitation, vol. 12, no. 10, pp. 805-823, 1980. 

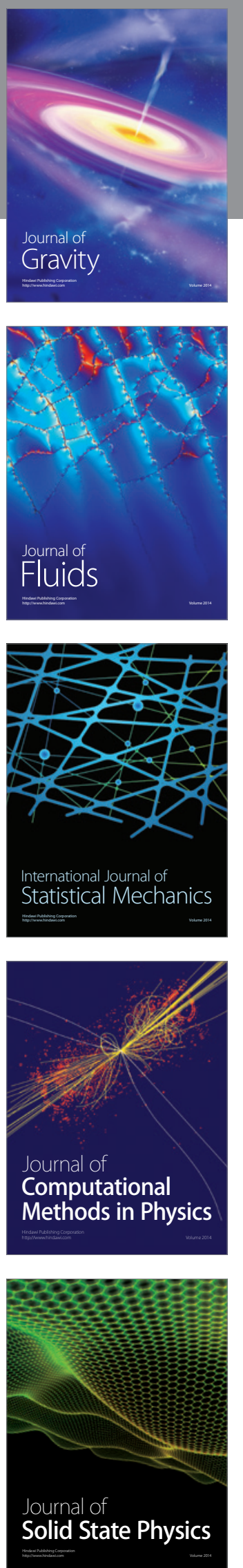

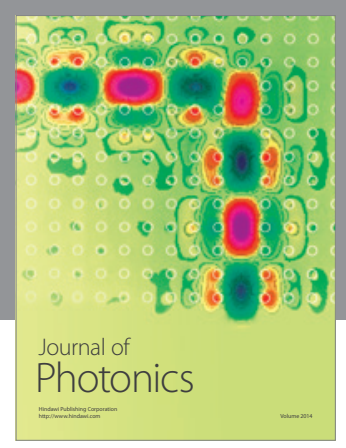

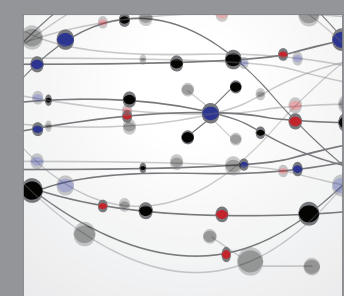

The Scientific World Journal
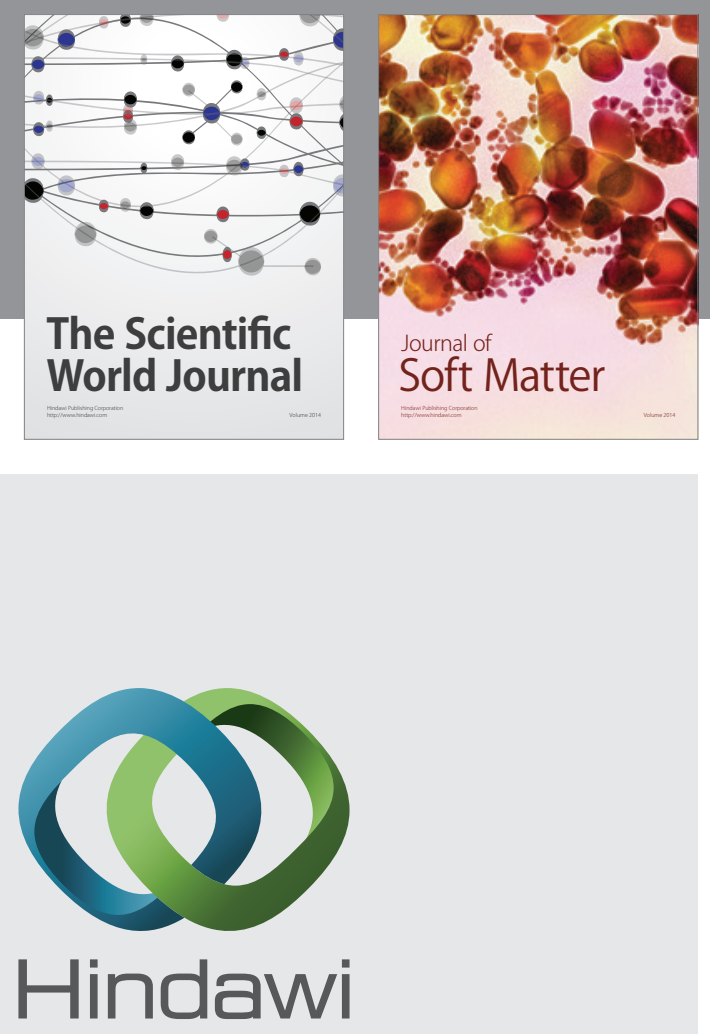

Submit your manuscripts at

http://www.hindawi.com
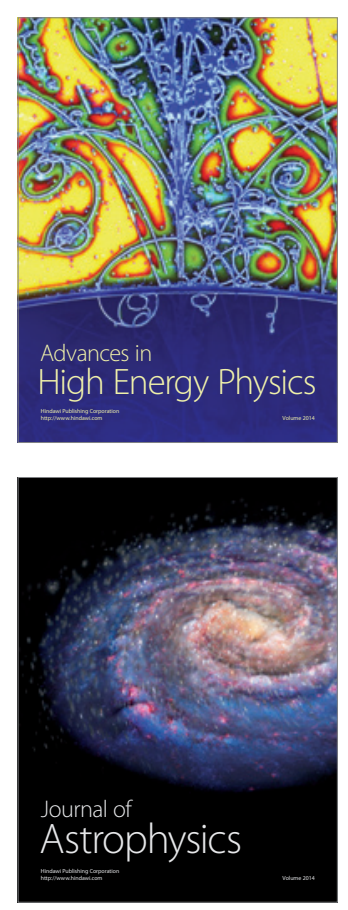
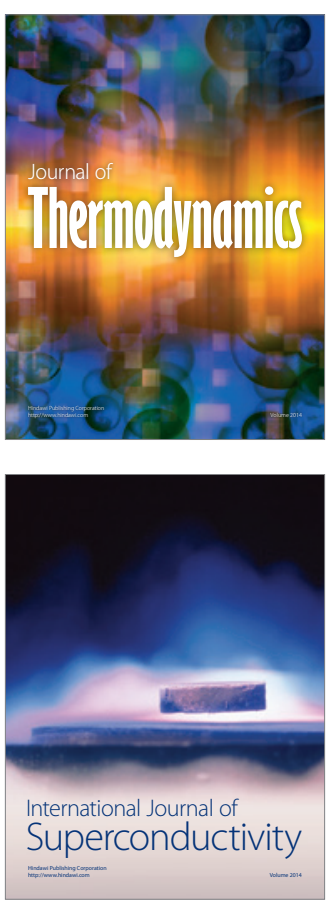
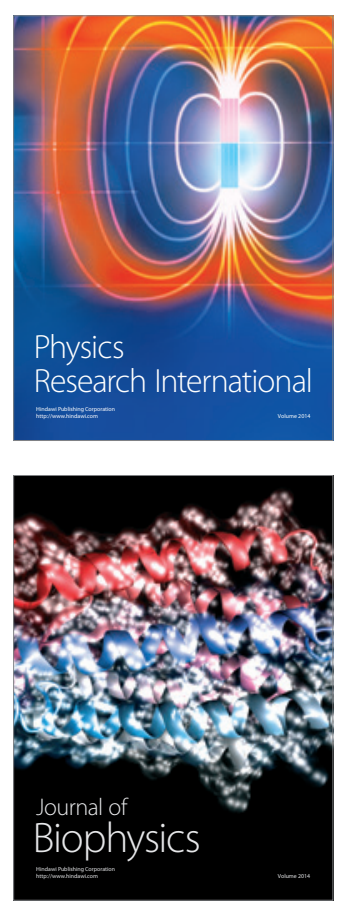
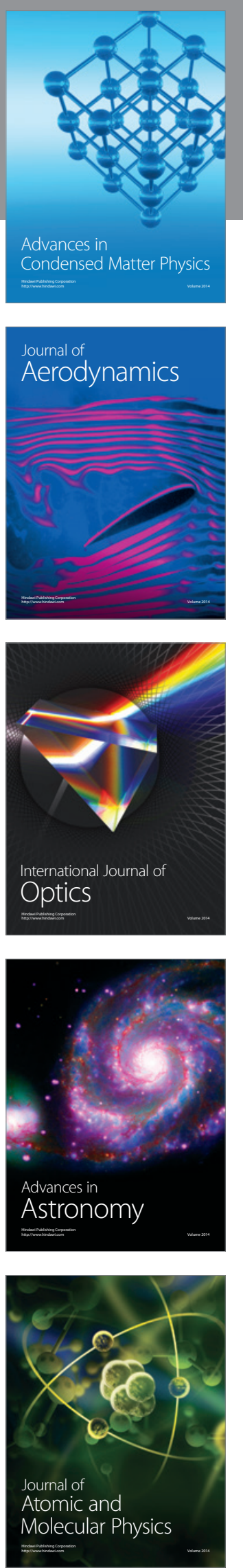